\title{
Laser, Universe and Arrow of Time
}

\author{
Mitali Konwar', Gauranga Dhar Baruah² \\ ${ }^{1}$ Moran College, Sivasagar, India \\ ${ }^{2}$ Centre for Laser and Optical Science, Tinsukia, India \\ Email:mitalikonwar@rediffmail.com
}

How to cite this paper: Konwar, M. and Baruah, G.D. (2019) Laser, Universe and Arrow of Time. Optics and Photonics Journal, 9, 127-139. https://doi.org/10.4236/opj.2019.98012

Received: April 3, 2019

Accepted: August 19, 2019

Published: August 22, 2019

Copyright (C) 2019 by author(s) and Scientific Research Publishing Inc. This work is licensed under the Creative Commons Attribution International License (CC BY 4.0).

http://creativecommons.org/licenses/by/4.0/

\begin{abstract}
We have presented a comparison between the universe and the Laser. In many ways, the physics of laser and the universe are analogous. The root of the analogy is the fact that both laser and early universe depend completely on the quantum nature. We have also presented a simple analogous example of the growth of a flower at successive stages of development and shown how the arrow of time may be represented in these cases.
\end{abstract}

\section{Keywords \\ Laser, Universe, Time}

\section{Introduction}

Let us begin our article by reflecting on the truth of the universe, that is, the universe is of four dimensions (length, breadth, height and time). The immediate question is "why Einstein had formulated the theory". There are various ways to answer it. One way of answering is that-light waves of all frequencies travel with a constant speed in vacuum. It is surprising and unexpected that this speed is independent of relative speeds of the source and observer. This fact led Einstein to formulate special theory of relativity and concluded that space and time are separate entities but constitute the part of the same space-time continuum of a four-dimensional universe. As Einstein writes in his revolutionary paper [1] "Together with the unsuccessful attempts to discover any motion of the earth relatively to the 'light medium' suggest that the phenomena of electrodynamics as well as of mechanics posses no properties corresponding to the idea of absolute rest". What they did suggest, he went on, was that "The same law of electrodynamics and optics will be valid for all frames of reference for which the equations of mechanics hold good". Linking of electrodynamics and mechanics was the crux of matter. In the world of electromagnetism governed by the field laws Faraday 
and Maxwell, light was propagated at a constant speed which could not be surpassed. But there seemed to be little connection here with Newtonian mechanics, in which the speed of an object might be indefinitely increased by adding more energy to it. What Einstein proposed in his paper was that the velocity of light is a constant and a maximum in the electromagnetic and mechanical worlds and that light would thus travel with a constant velocity that was independent of the bodies emitting or receiving it. Minkowski's contribution to the development of special relativity was in effect a single paper "Basic Equations for the Electromagnetic phenomena in moving bodies" published in 1907 [2]. Minkowski not only gave a new mathematical formulism to the special theory but also enabled Einstein to solve the problems of gravitation by means of general relativity. Thus according to Minkowski an event which takes place in three-dimensional space at a particular time is described as a "world point" described as a "world line". More significantly time is described as a "fourth dimension". Einstein was well aware of the bewilderment which such language created. "The non-mathematician 'he wrote' is seized by a mysterious shuddering when he hears of 'four-dimensional thing' by feeling not unlike that awakened by thoughts of the occult. And yet there is no more common place statement than that the world which we live in is a four-dimensional space time continuum" signifier for a mathematician a fourth variable apart of length, breadth or thickness which must be inserted into any equation concerning events, since these occur not only in space but at a certain instant of time.

The purpose of the present paper is to introduce the topic of the direction of time or arrow of time as discussed by Hawking [3] and to understand it in some environmental and non physics domain. In this paper we are primarily concerned with a familiar topic of a growth of a flower in its successive stages of development commencing from the tender bud which has a greenish hue and proceeding by steps to the mature flower exhibiting a full colour and how the so called arrow of time as discussed by Hawking may be represented and understood. We have also discussed the role of a so called spectral line in understanding this arrow. It is worthy of remark here that one way of looking at a problem is from analogy. In many ways some phenomena in physics appearing in different domains are quite analogous. There are numerous examples to support this. One example is the comparison between laser near threshold and matter near a phase transition as developed by Degiorgio and Scully [4]. Recently we have worked out few analogies which cover specific topics like spatial hole burning, multiple reflection and squeezed states [5], dispersion curves of semi-classical theory of laser and electromagnetically induced transparency [6], analogies between spectroscopic properties of laser and the universe [7]. In this paper we carry the analogies further and introduce additional features so that a worthwhile comparison with the properties of the universe may be made. It is worthy of remark that when a problem is compared with something identical in a different field but which one understands reasonably comparative ideas are created 
and this is one way of looking at the problem.

The paper is organized as follows. Section 2 will be a brief review of the topic of arrow of time as discussed by Hawking. The coherent properties of Laser radiation are discussed in Section 3. In Section 4 we have brought under discussion the topics of the quantum theory of radiation, vacuum fluctuation, quantum theory of Laser and quantum cosmology. These topics have been primarily chosen for a worthwhile comparison between laser and the evolution of the quantum universe as shown in Table 1. In Section 5 we have presented the specific example of a flower in the successive stages of development commencing from the tender bud which has a greenish tint and proceeding by steps to the

Table 1. Laser, arrow of time and the evolution of the universe.

\begin{tabular}{|c|c|c|}
\hline Parameter & Universe & Laser \\
\hline Entropy & $\begin{array}{l}\text { As the universe is expanding (and currently } \\
\text { accelerating) entropy increases }\end{array}$ & Entropy increases \\
\hline Time & $\begin{array}{l}\text { According to Hawking the universe we live in } \\
\text { does not appear time symmetric }\end{array}$ & Laser is not time symmetric \\
\hline Time & $\begin{array}{l}\text { The thermodynamic arrow of time, the } \\
\text { psychological arrow of time and the } \\
\text { cosmological arrow of time all point in the } \\
\text { same direction. These arrows explain } \\
\text { the time asymmetry of the universe }\end{array}$ & $\begin{array}{l}\text { Thermodynamic arrow of } \\
\text { time is directed along the } \\
\text { laser axis }\end{array}$ \\
\hline Origin & $\begin{array}{l}\text { Origin of the universe took place } \\
\text { as a "Big Bang" }\end{array}$ & $\begin{array}{l}\text { Laser action takes place at a } \\
\text { threshold condition of } \\
\text { oscillation }\end{array}$ \\
\hline Origin & $\begin{array}{l}\text { For some reason the universe started } \\
\text { out in a stable of high order (low entropy) }\end{array}$ & $\begin{array}{l}\text { Laser started out in a state of } \\
\text { high order (population } \\
\text { inversion) }\end{array}$ \\
\hline Temperature & $\begin{array}{l}\text { Temperature at a time of } 10^{-12} \text { sec. after Big } \\
\text { Bang is estimated at } 10^{150} \mathrm{~K}\end{array}$ & $\begin{array}{l}\text { Laser output can be described } \\
\text { as having an effective } \\
\text { temperature of } \sim 10^{180} \mathrm{~K}\end{array}$ \\
\hline Space-time & $\begin{array}{l}\text { In special theory an event occurs not only in } \\
\text { space but also in time. They are known as } \\
\text { world points and linking events are world link }\end{array}$ & $\begin{array}{l}\text { Laser radiation is generally } \\
\text { space and time coherent }\end{array}$ \\
\hline $\begin{array}{l}\text { Vacuum } \\
\text { fluctuation }\end{array}$ & $\begin{array}{l}\text { In quantum theory of cosmology the universe } \\
\text { can be created from nothing spontaneously by } \\
\text { virtue of vacuum fluctuations }\end{array}$ & $\begin{array}{l}\text { The mechanism of } \\
\text { spontaneous emission is } \\
\text { attributed in connection with } \\
\text { quantum theory to all } \\
\text { pervading vacuum } \\
\text { fluctuations of the } \\
\text { electromagnetic field. Laser } \\
\text { photon also builds up from } \\
\text { vacuum fluctuations }\end{array}$ \\
\hline $\begin{array}{l}\text { Exponential } \\
\text { growth }\end{array}$ & $\begin{array}{l}\text { In the quantum theory of cosmology when } \\
\text { the true vacuum bubble expands rapidly } \\
\text { the universe can be created irreversibly }\end{array}$ & $\begin{array}{l}\text { In the quantum theory of } \\
\text { laser photon grows } \\
\text { exponentially in the initial } \\
\text { stage as it is created from } \\
\text { vacuum }\end{array}$ \\
\hline
\end{tabular}


mature flower exhibiting full colour. We have represented thermodynamic arrow of time in these cases and we believe that such examples even as analogy is relevant for science education.

\section{Arrow of Time}

Before we introduce the topic of arrow of time as introduced by Hawking, let us first consider the question: which is the "real" time and which is the "real" dimension. The answer is simple. The real dimension and the real time is that of the observer, and the stationary and the moving observers are each concerned with their own reality. Each man carries with his own space and his own time. The time at which an event takes place is indeed a relative matter. It is now worthwhile to consider the problem of the direction of time in cosmology as discussed by Hawking. There are two things Hawkings emphasized. One is that physics is time symmetric; the second is that the universe that we live in certainly does not appear time symmetric. That physics is time symmetric is more accurately shown that [8] any quantum field that has 1) Lorentz invariance, 2) Positive energy and 3) Local causality, i.e. $\emptyset(x)$ and $\emptyset(y)$ commute (or anti-commute) if $\mathrm{x}$ and y are space like separated, is invariant under CPT. Here $C$ means interchange particles with antiparticles, $P$ means replace left hand by left hand, and $\mathrm{T}$ means reverse the direction of motion of all particles. In most situations, the effect of any $C$ or $P$ non-variance can be neglected, so that the interactions ought to be invariant under $T$ alone. In his work Hawking indicates that the universe that we live in certainly does not appear time symmetric, as anymore who has watched a movie being shown backward can testify: one sees events that are not witnessed in ordinary life. One can identify a number of different "arrows of time" that express the time asymmetry of the universe. The thermodynamic arrow: the direction of time in which entropy increases. The electro-dynamic arrow: the fact that one uses retarded solutions of the field equations rather than the advanced one. The psychological arrow: this arrow of time is the way time appears to flow to the human mind (real time) - the way we experience the unceasing aging of our bodies, remember the past and have no direct knowledge of the future. The cosmological arrow: the direction in time in which the universe is expanding (currently accelerating) from big bang. It is worthy of remark here that we have introduced here in our paper an arrow of time which we call as the local arrow of time. According to Hawking the first arrow of time implies the second and third. The connection between the psychological and thermodynamic arrows can be discussed from an analogy with computers; when a computer records something in the memory, that is, when it records digitally in bits and q-bits (quantum bits), the total entropy increases. Thus computer remembers things in the direction of time in which the entropy increases. Thus it seems reasonable to assume that we remember in the same direction of time that computers do. This means that the psychological arrow of time, our subjective sense of time, is the same as the thermodynamic arrow of 
time, the direction in which entropy increases. Entropy increases with time because we define the direction of time to be that in which entropy increases [9]. What was the connection between the cosmological arrow of time and others? Hawking put forth the controversial conjecture that although the three arrows all point in the same direction during the current expanding phase of the universe, he believed that the no boundary proposal (which utilised closed universe doomed to eventually collapse in on themselves) would predict that when the universe began to contract, the thermodynamic arrow of time (and so by the previous arguments the psychological arrow of time as well) would reverse. He proposed that entropy would decrease rather than increase, meaning that things could became more ordered rather than random. Hawking's proposal would also mean that people will grow younger rather than older. We would remember the future and have no knowledge of the past. However Hawking finally admitted that he had been wrong about the reversal of the thermo-dynamical and psychological arrow of time in a contracting universe. Hawking and Collins [10] connected the fact that the overall distribution of matter in the universe is remarkably similar in all direction we observe (isotropic) to the density perturbations in the early universe (which led to the creation of galaxies) and found that the balance to be highly unlikely. They came to the conclusion that we have observed the universe to be isotropic is therefore only a consequence of our own existence. If it were otherwise galaxies would not exist. With these concepts in mind Hawking pondered the problem of the three arrows of time and came to the conclusion that although the psychological and thermo dynamic arrows did not reverse during the contracting phase of the universe, it is only when the three arrows of time pointed in the same direction the intelligent life could exist who could contemplate the problem. In this connection it is worthwhile to mention the work of Hogarth [11] and Hoyle and Narlikar [12] in the early 1960's when they tried to connect the electro dynamic and cosmological arrows. Richard Feynman [13] criticized their work on the grounds that they had implicitly assumed the electro dynamic arrow. They also got the wrong answer in that they predicted retarded potentials in a steady state universe without continual creation of matter. According to Hawking it is generally accepted that we live in a universe which is still evolving. It is now worthwhile to close this section with a remark that the relevant mathematics which concerns the arrows of time are more involved. We shall concern ourselves in the following sections with a more elementary approach to represent arrows of time which we believe is helpful for science education particularly in understanding the arrow of time. We shall discuss few topics like a spectral line, the nature of growth in the successive development of flowers, laser, vacuum fluctuations etc. and see how analogous they are with our evolving universe.

\section{Coherent Properties of Laser Radiation}

In this section we specifically describe the coherent properties of laser radiation 
for a possible analogy with the world point and world line in the space time continuum of the special theory of relativity. We must indicate here that the topics set forth in the earlier sections motivated us to draw some analogy with the coherent properties of laser radiation, and to some degree of approximation a spectral line.

A laser radiation is both space and time coherent. A source of radiation may have little time coherence but still has perfect space coherence. Example of such coherent source is light from a distant star.

\subsection{Spatial Coherence}

If at a particular instant of time the radiation has the same phase across the uniphase wave front it is said to be completely spatial coherent. Laser beam moves in a particular direction is due to this property only.

\subsection{Temporal Coherence}

If the phase of the light of one uniphase wave front at a particular time is the same with it displaced wave front after it has travelled a distance in time $t$ and if the phase agrees with all time interval, the radiation is said to be perfectly time coherent. Extreme monochromatically is the manifestation of the property of temporal coherence.

\subsection{High Intensity}

The usual optical radiation has an intensity characterised by an effective temperature of the order of few thousands of degrees only, on the other hand the laser output can be described as having an effective temperature of $10^{180} \mathrm{~K}$ or more.

\section{Quantum Theory of Radiation, Vacuum Fluctuation, Quantum Theory of Laser and Quantum Cosmology}

The basic facts and principles relating to the special theory of relativity as formulated by Einstein [1] with the three arrows of time articulated by Hawking [3] have been expounded in earlier sections. We have also brought under discussion the topics of spectral line and coherent properties of laser radiation in order to correlate them with the topics of space time continuum and as well as the arrows of time. The correlations are presumably for analogy consideration only. We now consider it worthwhile to discuss the quantum theory of laser and the process of buildup of laser photon from vacuum as formulated by Lamb and co-workers. Laser is basically created inside a resonating cavity and as it emerges out of the partially reflecting dielectric mirror it appears as a unidirectional strong beam of monochromatic radiation coherence. Spontaneous emission is the driving force which acts as the platform for the laser radiation to be built up. The interesting consequence of the quantization of radiation field is the fluctuation associated with the zero-point energy or the so called vacuum fluctuations. 
These fluctuations are responsible for many interesting phenomena in quantum optics like spontaneous emission. The mechanism of spontaneous emission-an isotropic perturbation always presents theory to the all pervading zero point fluctuations of the electromagnetic field. The light excites the atoms, the zero point fluctuation de-excites them resulting in re radiation of light.

The role of spontaneous emission is emphasized in the quantum theory of laser by Lamb and co-workers [14] [15]. The buildup of laser photon takes place from spontaneous noise. To do this we show that the average photon number is given by

$$
\langle n(t)\rangle=\sum n P_{n n}(t)
$$

And it may be shown that the equation of motion for the average photon number $<n(t)>$ is given by

$$
\frac{\mathrm{d}}{\mathrm{d} t}\langle n(t)\rangle=\left(A-\frac{v}{Q}\right)\langle n\rangle+A-B\left[\left\langle n^{2}\right\rangle+2\langle n\rangle+1\right]
$$

where $A$ is the linear gain coefficient in quantum theory of laser, $V$ is the laser oscillation frequency in radian/Sec (not Hertz) and $Q$ is the cavity quality factor. The above equation has been used to investigate the process of build up laser photon from vacuum [16]. We note here that the lone term $A$ in the equation represents the spontaneous noise originating from vacuum fluctuations.

The accepted explanation for the thermo dynamic arrow of time is that for some reason the Universe started out in a state high order or low entropy. Such states occupy a very small fraction of volume of the phase space accessible to the universe. As the universe evolves in time it will tend to move around phase space ergodically. In this connection it is worthwhile to the Ergodic Hypothesis in statistical physics concerning "phase space". If a system of $\mathrm{N}$ atoms is enclosed in a fixed volume, the state of the system is given by a point is a $6 \mathrm{~N}$-dimensional phase space with $q$ representing coordinates and $E$ to be constant a representative point in phase space describe an orbit on the surface $E(q, p)=C$, where $\mathrm{c}$ is a constant. The ergodic hypothesis states that the orbit of the representative point in phase space eventually goes through all the points on the surface.

Thus at a later time as Hawking says, therefore there is a high probability that the universe will be found in a state of disorder or high entropy because such states occupy most of phase space. Consider again the example given by Hawking a system of $\mathrm{N}$ gas molecules in a rectangular box which is divided into two by a partition with a small hole in it. Suppose that some initial time, all the molecules are in the left hand side of the box. Such configurations occupy only one part in $2 \mathrm{~N}$ of the available $6 \mathrm{~N}$-dimensional phase space. As time evolves the system will move around phase space on a constant energy surface. At a later time there will be higher probability of finding the system in a more disordered state with molecules in both halves of the box. Thus entropy will increase with time. But if one waits long enough, one will find all the molecules eventually returning 
to one half of the box. However, for macroscopic values of N, the time taken is infinitely large and is likely to be much longer that the age of the universe. This is presumably the consequence of ergodic hypothesis. In general it is very difficult to prove the ergodic hypothesis. In this connection we may also refer to the so called KAM theorem (named after Russian mathematician A. M. Kolmogorov and V. I. Arnold and the German mathematician J. Maser) which states that a non-negligible fraction of orbits in the Phase space of a dynamical system remain indefinitely in a specific region of phase space, even in the presence of small perturbations of the system. This results in a step towards resolving the unsolved problem of the stability of motion of planets. The KAM theorem is also of interest to ergodicity in statistical mechanic, since the majority of orbits lead to ergodic motion going through all available phase space. As the number of degree of freedom of the system increases, the dominance of ergodicity over stable orbits becomes more pronounced.

In connection with the phenomenon of laser we note that one of the criteria of laser is the population inversion, which is the violation of usual Boltzman distribution and a highly ordered situation similar to the example given b Hawking regarding all the molecules being located in the left hand side of a rectangular box (divided into two parts by a partition with a small hole in it) at a particular time. We reasonably believe that entropy will increase with time as laser beam propagates through space. This is due to the fact that the concepts of space and time coherency will not be valid for any practical laser after it has travelled a huge distance resulting a increase in disorder. Thus entropy increases and direction of laser is the direction of the thermodynamic arrow of time. How the entropy increases as the laser beam propagates can be visualised in terms of the distance through which the beam maintain the constant phase relationship. This length is known as the coherence length and is given approximately by the relationship.

$$
\ell=c / \Delta v
$$

where $c$ is the velocity of light and $\Delta v$ is the bandwidth of the source. Thus the reduction of laser frequency bandwidth significantly increases the coherence length or coherence time. As the laser radiation travels through space, the beam spread increases which are also a measure of increase of disorder (entropy).

Now it is worthwhile to speculate that laser started out in a state of high order or low entropy which is analogous to the evolution of the universe currently expanding (or accelerating) from the big bang. We not here that the temperature at a time of $10^{-12}$ second from big bang is estimated to be at $10^{150} \mathrm{~K}$ (present day microwave cosmic background correspond to about $2.7^{0} \mathrm{~K}$ ). The laser output can also be described as having an unbelievable high value of $10^{180} \mathrm{~K}$ or more. The time to achieve lasing action from the time optional (or electrical) pumping is applied on the active medium inside a F P cavity is around $10^{-9}$ seconds, which depends on the length of the cavity.

That the universe began in a big bang is one of the great theories of modern 
cosmology. This theory is presumably a scientific theory supported by numerous evidences. But these still remains an enigma. What caused the big bang itself. For many years cosmologists have relied in the idea that the universe formed spontaneously that the big bang was the result of quantum fluctuations in which the universe came into existence from nothing. Tryon [17] published a paper in Nature where he proposes a model to support the theory that our universe is a large scale fluctuation of vacuum, where "vacuum fluctuation" is to be understood in the sense of quantum field theory. In a recent paper of 2014 Dongsham et al. [18] showed rigorously that the birth of the early universe from big bang could indeed have occurred spontaneously because of quantum fluctuations. How Dongsham et al. gave a rigorous proof of the idea that the universe could be spontaneously created from nothing can be briefly described as follows. According to them with the Lamda-cold dark matter (ACDM) model and with all available observation such cosmic microwave background, abundance of light elements, it has been widely accepted that the universe was created in a big bang. However there are still some puzzles, such as problems of flatness, the horizon, the monopole and the singularity [19]. In particular, the inflation theories which suggest that the universe experienced an exponential expansion period were proposed to solve puzzles of the early universe [20] [21] [22]. In quantum cosmology the universe is described by a wave function rather than the classical space time. The wave function of the universe should satisfy the Wheeler-Dewitt equation (WDWE) [23].

With the development of quantum cosmology theory, it has been suggested that the universe can be created spontaneously from nothing where nothingness means there is neither matter nor space or time [24], and the problem of singularity can be avoided naturally. Although the concept of the universe created spontaneously from nothing has emerged for a long time, a rigorous mathematical foundation for this is still missing. According to Heisenberg uncertainty principle, a small empty space also called a true vacuum bubble can be created by quantum fluctuations of the metastable false vacuum.

But if the small bubble cannot expand rapidly, it will disappear soon due to quantum fluctuations. In this case the early universe would disappear before it grows up. On the other hand if the small bubble expands rapidly to a large enough size. In 2014 paper Dongshan et al. obtained analytical solution of the WDWE of the true vacuum bubble. With the de Broglie-Bohn quantum trajectory they prove that once a small true vacuum bubble is created, it has the chance to expand exponentially when it is very small, that is $a \ll 1$ where a is the radius of the bubble.

The exponential expansion will end when the size of the true vacuum bubble is very large, that is, $a \gg 1$. It is also shown that the quantum potential of the bubble is the driving force for the exponential expansion and it also plays the role of cosmological constant.

This explicitly shows that the universe can be created spontaneously by virtue of a quantum mechanism. 
We now feel it appropriate to use Table 1 to include all the considerations set forth in earlier sections so that a summary of the comparisons between laser radiation and the evolution of the universe may be presented. In Table 2 of the following Section 6, we show specifically how the arrow of time may be represented in the successive stages of development of flowers (as analogy).

\section{Successive Stages of Development of Flowers}

It is quite instructive of examine the flower of a particular plant in the successive stages of development commencing from the tender bud which has a greenish tint and proceeding by steps to the mature flower exhibiting full colour. We may represent thermodynamic arrow of time in these cases at least as analogy, and which we believe is relevant for science education. For the purpose of illustration we have reproduced the photographs of few specimens of flowers at different stages of growth occurring at different times. It is worthwhile to note that we could have selected numerous other examples for the purpose of our illustration, but the consideration set forth in the present case is also applicable in these examples (Figure 1).

Figure 1 shows the photographs of a flower chrysanthemum at successive stages of development. The photographs have been taken with the help of a smart phone after every twelve hours to investigate its nature of growth in time. As may be inferred from the photographs, the linear growth of the flower takes place from some non-zero value and after some time becomes maximum and remains constant. The situation is well illustrated with the help of Figure 2 where we have plotted the linear dimension of the flower (in $\mathrm{cm}$ ) against time. For every twelve hours the measurements have been made. As may be inferred from the figure the growth of the flower initially indicates exponential growth from some non-zero value, after which there is a rapid growth in linear dimension

Table 2. Summary of the characteristic features in the successive development of flowers (events).

\begin{tabular}{cll}
\hline Quantity & \multicolumn{1}{c}{ Event } & \multicolumn{1}{c}{ Remark } \\
\hline Entropy & $\begin{array}{l}\text { Entropy increases as the flower grows } \\
\text { in size at different time }\end{array}$ & $\begin{array}{l}\text { Arrow of time is directed along } \\
\text { the direction of the increase in } \\
\text { disorder }\end{array}$ \\
Symmetry & $\begin{array}{l}\text { Successive stages of growth is } \\
\text { asymmetric in Nature }\end{array}$ & $\begin{array}{l}\text { The universe that we live in does } \\
\text { not appear time symmetric }\end{array}$ \\
process & $\begin{array}{l}\text { The flower initially builds up from a } \\
\text { tender bud (non zero value) indicating } \\
\text { some sort of Fluctuation }\end{array}$ & $\begin{array}{l}\text { That the universe is build up } \\
\text { spontaneously from vacuum } \\
\text { fluctuation is shown in a recent } \\
\text { publication [19] }\end{array}$ \\
& & $\begin{array}{l}\text { The theory [19] indicates that } \\
\text { Irreversible, grows exponentially } \\
\text { in the initial stage }\end{array}$
\end{tabular}




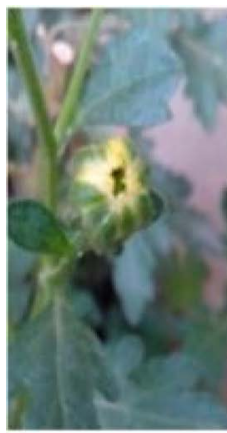

(a) Start

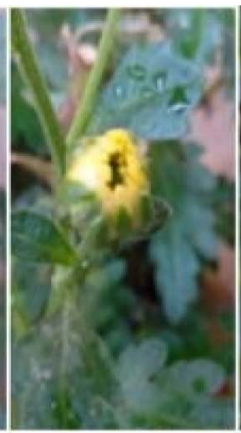

(b) $12 \mathrm{Hrs}$

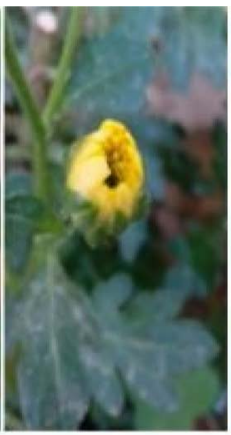

(c) $24 \mathrm{Hrs}$

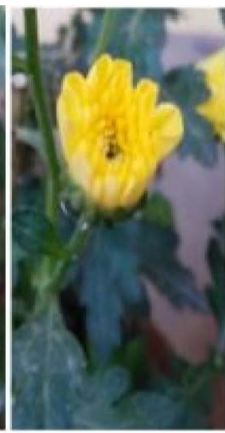

(d) $36 \mathrm{Hrs}$

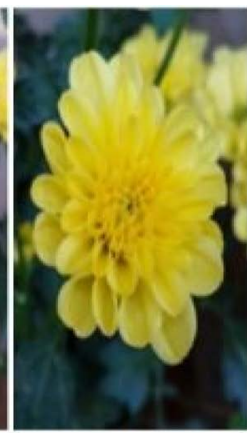

(e) $48 \mathrm{Hrs}$

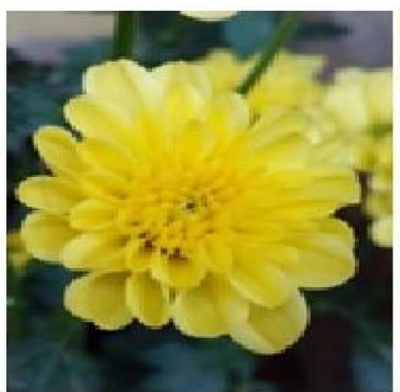

(f) $60 \mathrm{Hrs}$

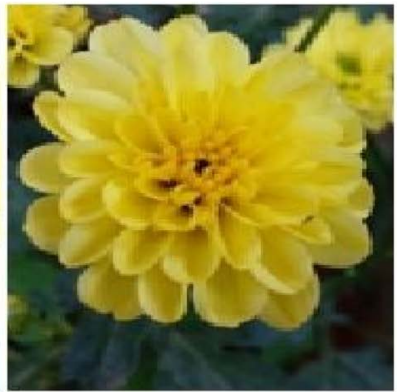

(g) $72 \mathrm{Hrs}$

Figure 1. Photograph of Chrysanthemum (Dendranthema grandiflora) flowers at successive stages of development.

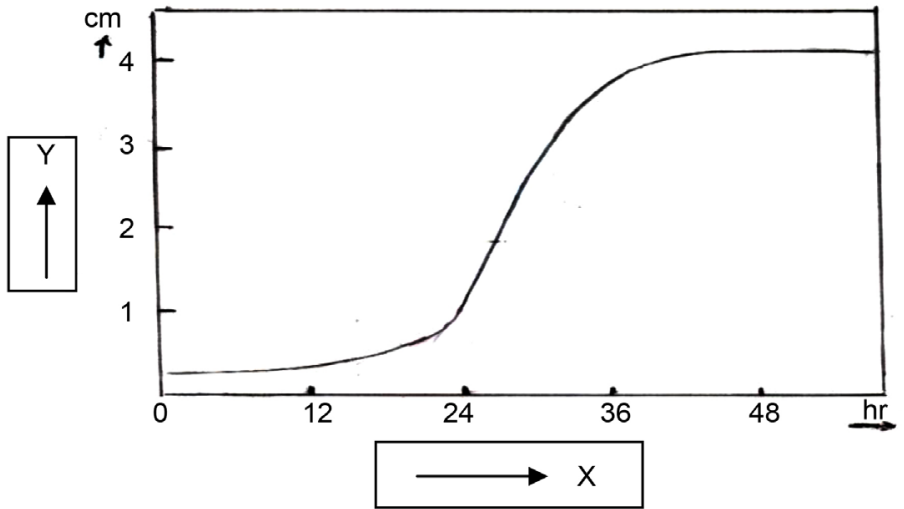

Figure 2. Build up of linear dimension of a flower Chrysanthemum (Dendranthema grandiflora) at successive stages (12 hr Interval of development).

and it yields steady state value after 72 hours. It is worthy of remark that this topic of graphs are quite common in biological specimens. We use the middle part of the growth curve to represent the arrow of time, which we designate as local arrow of time. It is quite interesting to use the following equation to represent the growth of a flower at successive stages of development (or any biological specimen). It is of the form:

$$
I_{n}(t)=I_{n}(t)=\frac{I_{n} I_{0} \exp (2) a_{n} t}{I_{n}-I_{0}+I_{0} \exp \left(2 a_{n} t\right)}
$$

where $I_{n}$ is the linear growth of the flower at its maximum $I_{0}$ is the linear growth 
of the specimen at a time before onset of threshold growth. It must be emphasized that $I_{0}$ is always finite. This is similar to the parameter $I_{\mathrm{n}}(0)$ in the semi classical theory of laser. We treat the equation as empirical as it is not derived rigorously like the intensity equation of motion and its solution in the semi classical theory. Now we include all the considerations set forth in this section in a tabular form so that some of the characteristic features of the arrow of time may be visualized.

\section{Summary and Conclusion}

We have presented a comparison between the universe and the laser. Some topics which are quite analogous are included in Table 1. It must be emphasized that the characteristic features as shown for laser and the universe are already well established in the literature. As may be inferred from Table 1 in many ways, the physics of the laser is analogous to that of the evolution of the universe. The root of the analogy is the fact that both the laser and the early universe depend completely on the quantum nature of the theory. We have also presented a simple example of the growth of a flower in the successive stages of development and show how the arrow of time may be represented in these cases.

\section{Conflicts of Interest}

The authors declare no conflicts of interest regarding the publication of this paper.

\section{References}

[1] Einstein, A. (1905) Zur Elektrodynamik bewegter Korper. Annalen der Physik, 17, 891-921.

[2] Minkowski, H. (1907) Gotinger Nachrichten.

[3] Hawking, S.W. (1985) Arrow of Time in Cosmology. Physical Review D, 32, 2489-2495.

[4] DeGiorgio, V. and Scully, M.O. (1970) Analogy between the Laser Threshold Region and a Second-Order Phase Transition. Physical Review A, 2, 1170. https://doi.org/10.1103/PhysRevA.2.1170

[5] Saikia, J., Dubey, R.K. and Baruah, G.D. (2015) Squeezed State Of Light, Multiple Reflection and Spatial Hole Burning. Journal of Multi-Disciplinary Engineering Technologies, 2, 2968.

[6] Bora Bordoloi, R., Bordoloi, R. and Baruah, G.D. (2015) Analogies between Spectroscopic Properties of Laser and the Universe. JMul. Eng. Sci. Tech, 2, 2851.

[7] Bora Bordoloi, R., Bordoloi, R., Saikia, J., Konwar, H. and Baruah, G.D. (2016) Analogies between Spectroscopic Properties of Laser and the Universea. Paripex-Indian Journal of Research, 5, 138.

[8] Konwar, M., Dewri, P.P. and Baruah, G.D. (2017) Arrow of Time in the Successive Stages of Development of Flowers. Int. J. Sci. Res. Eng, 2, 1.

[9] Streater, R.F. and Wightmann, A.S. (1964) PCT, Spin, Statistics and All That. Benjamin, New York.

[10] Hawking, S.W. (1992) The No Boundary Proposal and the Arrow of Time. In: Halliwell, J.J., Perez Mercader, J. and Zurek, W.H., Eds., Physical Origin of Time 
Asymmetry, Cambridge University Press, Cambridge, 268.

[11] Collins, C.B. and Hawking, S.W. (1973) Why Is the Universe Isotropic? Astrophysical Journal, 180, 317-334. https://doi.org/10.1086/151965

[12] Hogarh, J.E. (1962) Cosmological Considerations of the Absorber Theory of Radiation. Proceedings of the Royal Society of London. Series A, 267, 365. https://doi.org/10.1098/rspa.1962.0105

[13] Hoyle, F. and Narlikar, J.V. (1964) Time Symmetric Electrodynamics and the Arrow of Time in Cosmology. Proceedings of the Royal Society of London, Series A, 277, 1-23. https://doi.org/10.1098/rspa.1964.0002

[14] Gold, T. and Schumacher, D.L. (1967) The Nature of Time. Cornell University Press, Ithaca.

[15] Scully, M.O. and Lamb Jr., W.E. (1967) Quantum Theory of an Optical Maser. I. General Theory. Physical Review, 159, 208. https://doi.org/10.1103/PhysRev.159.208

[16] Sargent III, M., Scully, M.O. and Lamb Jr., W.E. (1974) Laser Physics. Addition Wesley Publishing Company, Reading.

[17] Scully, M.O., Kim, D.M. and Lamb Jr., W.E. (1970) Quantum Theory of an Optical Maser. IV. Generalization to Include Finite Temperature and Cavity Detuning. Physical Review A, 2, 2529. https://doi.org/10.1103/PhysRevA.2.2529

[18] Tryon, E.P. (1973) Is the Universe a Vacuum Fluctuation? Nature, 246, 396-397. https://doi.org/10.1038/246396a0

[19] He, D., Gao, D. and Cai, G. (2016) Spontaneous Creation of the Universe from Nothing. Physical Review D, 89, Article ID: 083510. https://doi.org/10.1103/PhysRevD.89.083510

[20] Strobinsky, A.A. (1979) Spectrum of Relict Gravitational Radiation and the Early State of the Universe. Pis'ma Zh. Eksp. Teor. Fiz, 30, 719-723.

[21] Strobinsky, A.A. (1980) A New Type of Isotropic Cosmological Models without Singularity. Physics Letters B, 91, 99-102. https://doi.org/10.1016/0370-2693(80)90670-X

[22] Guth, A.H. (1967) Inflationary Universe: A Possible Solution to the Horizon and Flatness Problems. Physical Review D, 23, 347. https://doi.org/10.1103/PhysRevD.23.347

[23] DeWitt, B.S. (1967) Quantum Theory of Gravity. I. The Canonical Theory. Physical Review, 160, 1113-1148. https://doi.org/10.1103/PhysRev.160.1113

[24] Vilenkin, A. (1994) Approaches to Quantum Cosmology. Physical Review D, 50, 2581-2594. https://doi.org/10.1103/PhysRevD.50.2581 